\title{
Difference in Gastrointestinal Risk Associated with Use of GLP-I Receptor Agonists: A Real-World Pharmacovigilance Study
}

\author{
Yu Zhou' \\ Mingyu Chen' \\ Libin Liu ${ }^{2}$ \\ Zhou Chen' \\ 'Department of Clinical Pharmacy and \\ Pharmacy Administration, School of \\ Pharmacy, Fujian Medical University, \\ Fuzhou, People's Republic of China; \\ ${ }^{2}$ Department of Endocrinology, Fujian \\ Medical University Union Hospital, \\ Fuzhou, People's Republic of China
}

Correspondence: Zhou Chen; Libin Liu Tel +86-59l-22862587; $+86-591-86218562$

Email chenzhou@fjmu.edu.cn;

libinliu@fjmu.edu.cn
Background: Glucagon-like peptide-1 (GLP-1) receptor agonists (GLP-1RAs) are promising weight-loss drugs, but real-world data concerning the liability of GLP-1RAs in gastrointestinal safety are lacking. We examined the differences in gastrointestinal safety between semaglutide and liraglutide.

Materials and Methods: We used the US Food and Drug Administration (FDA) Adverse Event Reporting System (FAERS) database and retrieved data during the first three years of semaglutide and liraglutide approved by the FDA. Thirteen main gastrointestinal adverse drug reactions (GADRs) were evaluated. Patient demographics, treatment information, and outcome of events were summarized. Disproportionality analyses were conducted by estimating the reporting odds ratios (RORs) and 95\% confidence intervals (CIs).

Results: In the reported cases of semaglutide $(n=2047)$ and liraglutide $(n=4175)$, semaglutide had a higher pooled ROR and later pooled time-to-onset median of GADRs compared with those of liraglutide (5.53, 95\% CI 5.23-5.85 vs 3.95, 95\% CI 3.81-4.10; 7 days, Q1-Q3: 0-48 vs 4 days, Q1-Q3: 0-34.5). The thirteen GADRs associated with these two GLP-1RAs showed a significant difference in the profile of reporting risk and time-to-onset.

Conclusion: GLP-1RAs produce a spectrum of distinct classes of GADRs. The individual properties of GADRs between semaglutide and liraglutide might enable incretin-based treatment of obesity to be "tailored" to the needs of each patient.

Keywords: GLP-1 receptor agonists, gastrointestinal adverse drug reactions, pharmacovigilance database

\section{Introduction}

Obesity is a global public-health problem. As of 2015, 2.2 billion are overweight or obese worldwide. ${ }^{1}$ Obesity is accompanied by multiple problems, such as reduced exercise capacity, worse quality of life, as well as increased susceptibility to metabolic, cardiovascular, or cerebrovascular diseases. ${ }^{1}$ An energy-restricted diet combined with aerobic exercise is the most efficacious treatment to achieve significant and sustained weight loss, but is difficult to adhere to. The vast majority of obese patients tend to select drugs that can elicit weight loss, but these agents generate disappointing efficacies or incur severe side-effects. ${ }^{2}$

The US Food and Drug Administration (FDA) has established strict evaluation criteria for new weight-loss drugs, they must: (i) elicit $>5 \%$ weight loss within 1 year; (ii) improve metabolic markers (blood pressure, blood lipid level, blood glucose level). ${ }^{3}$ Initially, glucagon-like peptide-1 (GLP-1) receptor agonists (GLP-1RAs) 
were approved for type-2 diabetes mellitus (T2DM) treatment. They not only have a significant effect on lowering of blood glucose levels, they also aid weight reduction, lower blood pressure, and improve lipid profiles, ${ }^{4,5}$ which undoubtedly meet the criteria for weight loss set by the FDA. Given the positive results in multiple clinical trials, ${ }^{6-8}$ in June 2021 the FDA approved semaglutide (Wegovy ${ }^{\circledR} ; 2.4 \mathrm{mg}$, s.c.) for long-term weight management in obese or overweight adults. This is the second GLP-1RA approved for weight loss after liraglutide (Saxenda ${ }^{\circledR} ; 3.0 \mathrm{mg}$, s.c.). However, the safety problems of GLP-1RAs for weight-loss drugs cannot be ignored. ${ }^{9}$

The most well-established toxicities from GLP-1RA therapy are gastrointestinal adverse drug reactions (GADRs). They include nausea, vomiting, diarrhea, and constipation, which were closely related to activation of central and peripheral GLP-1 receptors. ${ }^{10,11}$ However, a systematic analysis of the timing, spectrum, clinical characteristics, and outcomes of these GADRs has not been undertaken in a large number of patients after GLP1RAs were approved for marketing. Furthermore, whether there are differences in GADRs among different GLP1RAs is not known. It has been shown that a lack of detection and timely treatment for GADRs may lead to deterioration of already present gastrointestinal and renal diseases. ${ }^{12}$ This scenario poses concerns for clinicians when deciding the most appropriate and safe medication regimen for a patient who is at risk of GADRs.

FDA Adverse Event Reporting System (FAERS) is a large-scale post-marketing surveillance database. It contains many real-world data from various populations. FAERS can be used to analyze the reported correlation between drugs and adverse events, and to assess the riskbenefit status of drugs over time. Herein, we chose two GLP-1RAs approved by the FDA for weight loss or obesity, liraglutide and semaglutide, as target drugs to study. We aimed to characterize and compare GADRs of different GLP-1RAs captured in the real-world using FAERS. Thus, our analyses may provide information that could be helpful in choosing weight-loss agents from the class of GLP-1RAs when making decisions on "individualized" treatment.

\section{Materials and Methods}

\section{Data Source}

FAERS was used as the data source. This database collects reports of adverse events from pharmaceutical enterprises, medical staff, lawyers and consumers, and is updated quarterly. Although FAERS is a US database, it has global coverage and receives reports from the European Union and other non-US countries. Therefore, the size and global coverage of this open database make it particularly suitable for analysis of spontaneous-data reporting. In FAERS, adverse events are coded using preferred terms (PTs) in the Medical Dictionary for Regulatory Activities (MedDRA) terminology, which organizes terms into a hierarchy. FAERS is publicly available and anonymous, so approval and written informed consent were waived by the ethics review board of the Ethical Committee of Fujian Medical University.

\section{Study Design}

Each GLP-1RA was identified in FAERS by generic and brand names listed in the Drugs@FDA Database (www. accessdata.fda.gov/scripts/cder/daf/). To reduce the number of confounding factors, the search was performed using the words semaglutide/Ozempic and liraglutide/ Victoza. A cross-sectional case/non-case study (known as "disproportionality analysis") was undertaken in a subset of FAERS during the first three years of semaglutide (2018-2020) and liraglutide (2010-2012) approved the FDA. Target GLP-1RAs with a reported role coded as a "primary suspect" (PS) drug were evaluated for inclusion. Cases were identified as reports with at least one of the following PTs related to GADRs according to MedDRA 24.0: "nausea", "vomiting", "diarrhea", "constipation", "abdominal pain upper", "abdominal pain", "eructation", "abdominal discomfort", "abdominal distension", "flatulence", "dyspepsia", "gastrointestinal disorder", and "gastrooesophageal reflux disease". All other reports were considered to be non-cases. For example, if reports with nausea were considered to be cases, then all other reports were considered to be non-cases, including reports with other gastrointestinal events. Times-to-onset (ie, latency in the occurrence of a given ADR, expressed in days) was calculated as the difference between the date the event occurred and the start of therapy. A "serious outcome of an event" was defined as leading to one of the following: death; threat to life; hospitalization (initial or prolonged); disability necessitating intervention to prevent permanent impairment/damage; congenital anomaly; other serious events.

\section{Statistical Analyses}

After removing duplicates based on the primary identification of each case, GADRs were compared among two 
Table I Characteristics of Patients Who Suffered Gastrointestinal Adverse Drug Reactions When Using Semaglutide or Liraglutide

\begin{tabular}{|c|c|c|}
\hline & Semaglutide $(n=2047)$ & Liraglutide $(n=4 \mid 75)$ \\
\hline Age, years, median (QI-Q3) & $64(56-7 I)$ & $60(53-67)$ \\
\hline \multicolumn{3}{|l|}{ Sex, number (\%) } \\
\hline Female & I07I (52.32) & $2604(62.37)$ \\
\hline Male & $962(47.00)$ & $1295(31.02)$ \\
\hline Not reported & $14(0.68)$ & $276(6.6 I)$ \\
\hline \multicolumn{3}{|l|}{ Type of reporter, number. (\%) } \\
\hline Health professional & $858(41.91)$ & I35I (32.36) \\
\hline Non-health professional & I I $83(57.79)$ & $2815(67.43)$ \\
\hline Unknown & $6(0.29)$ & $9(0.22)$ \\
\hline \multicolumn{3}{|l|}{ Reporting country, number (\%) } \\
\hline First & US/I833 (89.55) & US/3754 (89.92) \\
\hline Second & $\mathrm{CA} / 54(2.64)$ & $\mathrm{GB} / 7 \mathrm{I}(\mathrm{I} .70)$ \\
\hline Third & $\mathrm{GB} / 33(1.6 \mathrm{I})$ & $\mathrm{JP} / 69$ (1.65) \\
\hline \multicolumn{3}{|l|}{ Outcome of event, number (\%) ${ }^{a}$} \\
\hline Death & $12(0.59)$ & $16(0.38)$ \\
\hline Life-threatening & $15(0.73)$ & $53(1.27)$ \\
\hline Hospitalization (initial or prolonged) & $294(14.36)$ & $491(11.76)$ \\
\hline Disability & $22(1.07)$ & $18(0.43)$ \\
\hline Required intervention to prevent permanent impairment/damage & $3(0.15)$ & $4(0.10)$ \\
\hline Congenital anomaly & I (0.05) & 0 \\
\hline Other serious events & $361(17.64)$ & $397(9.51)$ \\
\hline
\end{tabular}

Note: ${ }^{a} \mathrm{~A}$ report may have one or more outcomes.

Abbreviations: GLP-IRA, glucagon-like peptide-I receptor agonist; US, United States of America; CA, Canada; GB, Great Britain; JP, Japan.

GLP-1RAs in the first three years of FDA-approval. A reporting odds ratio (ROR) with $95 \%$ confidence interval (CI) was calculated to indicate disproportionality. Significant disproportionality was considered to be present if the lower bound of the $95 \%$ CI was $>1$. Although the ROR does not offer risk quantification or calculation of the prevalence, it gives an approximate indication of the clinical significance of the signal strength. ${ }^{13}$ A higher ROR suggested a stronger reporting association between the target GLP-1RA and a GADR. Processing and analyses of data were conducted using $\mathrm{R}$ 4.0.2 ( $\mathrm{R}$ Institute for Statistical Computing, Vienna, Austria).

\section{Results}

\section{Descriptive Analyses}

We identified 6222 cases with GADRs using liraglutide or semaglutide as the PS drug. Of these, 2047 cases were reported for semaglutide, and 4175 cases for liraglutide (Table 1). The median age for these cases was 64 (range, 56-71) and 60 (range, 53-67) years, respectively. More women than men tended to report GADRs for these two GLP-1RAs (52.32\% vs 62.37\%). Cases exposed to these two GLP-1RAs associated with GADRs were reported primarily by non-healthcare professionals (57.79\% vs $67.43 \%$ ), and the vast majority were from the USA (89.55\% vs $89.92 \%$ ). Hospitalization (initial or prolonged) and other serious events accounted for most of corresponding cases $(14.36 \%$ vs $11.76 \%$, and $17.64 \%$ vs $9.51 \%$, respectively), as the major serious outcomes of event.

\section{Analyses of Reporting-Risk Profiles}

Irrespective of semaglutide and liraglutide, the number of reported cases for nausea, vomiting, diarrhea, constipation, and abdominal pain upper were the top-five most prevalent (Table 2). Further disproportionality analyses of reporting risk showed that almost GADRs were reported with greater frequency for semaglutide and liraglutide compared with that for the full database background (Table 2). Semaglutide had a higher pooled ROR (5.53, 95\% CI 5.23-5.85) compared with that of liraglutide $(3.95,95 \%$ CI $3.81-4.10)$. The reporting-risk profile of assigned GADRs between semaglutide and liraglutide had a significant difference (Figure 1A). The top five of RORs for semaglutide were ranked as eructation (45.25, 95\% CI 38.44-53.28), flatulence (8.65, 95\% CI 7.22-10.37), nausea (7.14, 95\% CI 6.69-7.63), vomiting (6.71, 95\% CI 6.19-7.27), and constipation (5.42, 95\% CI 
Table 2 RORs for Gastrointestinal Adverse Drug Reactions Upon Use of Semaglutide or Liraglutide

\begin{tabular}{|c|c|c|c|c|c|c|}
\hline \multirow{2}{*}{$\begin{array}{l}\text { Gastrointestinal Adverse Drug } \\
\text { Reaction }\end{array}$} & \multicolumn{3}{|c|}{ Semaglutide } & \multicolumn{3}{|c|}{ Liraglutide } \\
\hline & $\begin{array}{l}\text { Cases, } \\
\text { Number }\end{array}$ & ROR & $95 \% \mathrm{Cl}$ & $\begin{array}{l}\text { Cases, } \\
\text { Number }\end{array}$ & ROR & $95 \% \mathrm{Cl}$ \\
\hline Nausea & 1158 & 7.14 & $(6.69,7.63)$ & 2543 & 5.63 & $(5.39,5.88)$ \\
\hline Vomiting & 688 & 6.71 & $(6.19,7.27)$ & 870 & 2.48 & $(2.3 \mathrm{I}, 2.65)$ \\
\hline Diarrhea & 608 & 3.65 & $(3.35,3.97)$ & 987 & 2.88 & $(2.70,3.07)$ \\
\hline Constipation & 304 & 5.42 & $(4.83,6.09)$ & 397 & 3.01 & $(2.72,3.32)$ \\
\hline Abdominal pain upper & 241 & 4.62 & $(4.06,5.26)$ & 389 & 2.58 & $(2.33,2.86)$ \\
\hline Abdominal pain & 197 & 3.36 & $(2.92,3.88)$ & 303 & 1.47 & $(1.32,1.65)$ \\
\hline Eructation & 155 & 45.25 & $(38.44,53.28)$ & 263 & 22.70 & $(19.95,25.84)$ \\
\hline Abdominal discomfort & $14 \mid$ & 2.93 & $(2.48,3.47)$ & 142 & 1.36 & $(1.15,1.61)$ \\
\hline Abdominal distension & 136 & 5.17 & $(4.36,6.13)$ & 145 & 2.09 & $(1.77,2.46)$ \\
\hline Flatulence & 121 & 8.65 & $(7.22,10.37)$ & 204 & 5.02 & $(4.37,5.78)$ \\
\hline Dyspepsia & 93 & 3.91 & $(3.19,4.80)$ & 229 & 2.65 & $(2.32,3.02)$ \\
\hline Gastroesophageal reflux disease & 67 & 3.05 & $(2.39,3.88)$ & 31 & 0.74 & $(0.52,1.05)$ \\
\hline Gastrointestinal disorder & 73 & 3.28 & $(2.6 I, 4.14)$ & 142 & 1.36 & $(1.15,1.61)$ \\
\hline Pooled $^{a}$ & 2047 & 5.53 & $(5.23,5.85)$ & 4175 & 3.95 & $(3.8 I, 4.10)$ \\
\hline
\end{tabular}

Note: ${ }^{a} A$ report may have one or more gastrointestinal drug adverse reactions.

Abbreviations: ROR, reporting odds ratio; GLP-IRA, glucagon-like peptide-I receptor agonist.

4.83-6.09). The top five of RORs for liraglutide were ranked as eructation $(22.70,95 \%$ CI 19.95-25.84), nausea (5.63, 95\% CI 5.39-5.88), flatulence (5.02, 95\% CI 4.37-5.78), constipation (3.01, 95\% CI 2.72-3.32), diarrhea $(2.88,95 \%$ CI 2.70-3.07).

\section{Analyses of Time-to-Onset Profiles}

Table 3 shows the time-to-onset of cases with semaglutideand liraglutide-associated GADRs. Semaglutide had a later pooled median time-to-onset of GADRs (7 days; Q1-Q3: 048), compared with that of liraglutide (4 days; 0-34.5). The time-to-onset profile of assigned GADRs between semaglutide and liraglutide had a significant difference (Figure 1B). GADRs that had a relatively late time-to-onset associated with semaglutide were abdominal pain (17.5 days; Q1-Q3: 0-59), vomiting (14 days; Q1-Q3: 0-53), diarrhea (14 days; Q1-Q3: 0-54), gastrointestinal disorder (14 days; Q1-Q3: 0-30.25), constipation (10 days; Q1-Q3: 0-63). GADRs that had a relatively late time-to-onset associated with liraglutide were gastrointestinal disorder (35 days; Q1-Q3: 13-219), abdominal pain (34.5 days; Q1-Q3: 4.25-89), abdominal discomfort (11 days; Q1-Q3: 0.5-71.5), gastroesophageal reflux disease (11 days; Q1-Q3: 0.5-71.5), dyspepsia (7 days; Q1Q3: 0-54.5), abdominal pain upper (6 days; Q1-Q3: 0-52).

\section{Analyses of Dosage Profiles}

To explore the relationship between dosage and GADRs, we undertook a subgroup analysis by the disproportionality method (Figure 2). The ROR (95\% CI) for semaglutide dosage (subcutaneous administration) significantly associated with GADRs was 8.00 (7.03-9.11) for $0.25 \mathrm{mg} /$ onceweekly (QW), $7.10(6.01-8.38)$ for $0.5 \mathrm{mg} / \mathrm{QW}$, and 5.34 (4.25-6.71) for $1 \mathrm{mg} / \mathrm{QW}$. The ROR (95\% CI) for liraglutide dosage (subcutaneous administration) significantly associated with GADRs was 5.60 (5.07-6.19) for $0.6 \mathrm{mg} /$ oncedaily (QD), 4.31 (3.84-4.83) for $1.2 \mathrm{mg} / \mathrm{QD}$, and 2.78 (2.463.14) for $1.8 \mathrm{mg} / \mathrm{QD}$.

\section{Discussion}

In this real-world study, we revealed that two GLP-1RAs approved by the FDA for weight loss were significantly associated with over-reporting of thirteen distinct categories of GADRs. Moreover, we observed a discrepancy between two GLP-1RAs in the reporting-risk profile and time-to-onset of ADRs.

In view of the risk of obesity to public health and because achieving and maintaining weight loss by changing lifestyle alone is difficult, pharmacological methods are needed to help some people lose weight. ${ }^{2}$ GLP-1RAs are promising pharmacotherapies for effective weight loss. ${ }^{14,15}$ The mechanism of weight loss underlying GLP1RA therapy may be related to: (i) reductions in appetite and hunger; (ii) lower preference for energy-dense foods; (iii) alteration in food-reward pathways; (iv) reduction of food craving; (v) improvement in eating control. ${ }^{16,17}$ The results of several clinical trials have shown that GLP- 
A

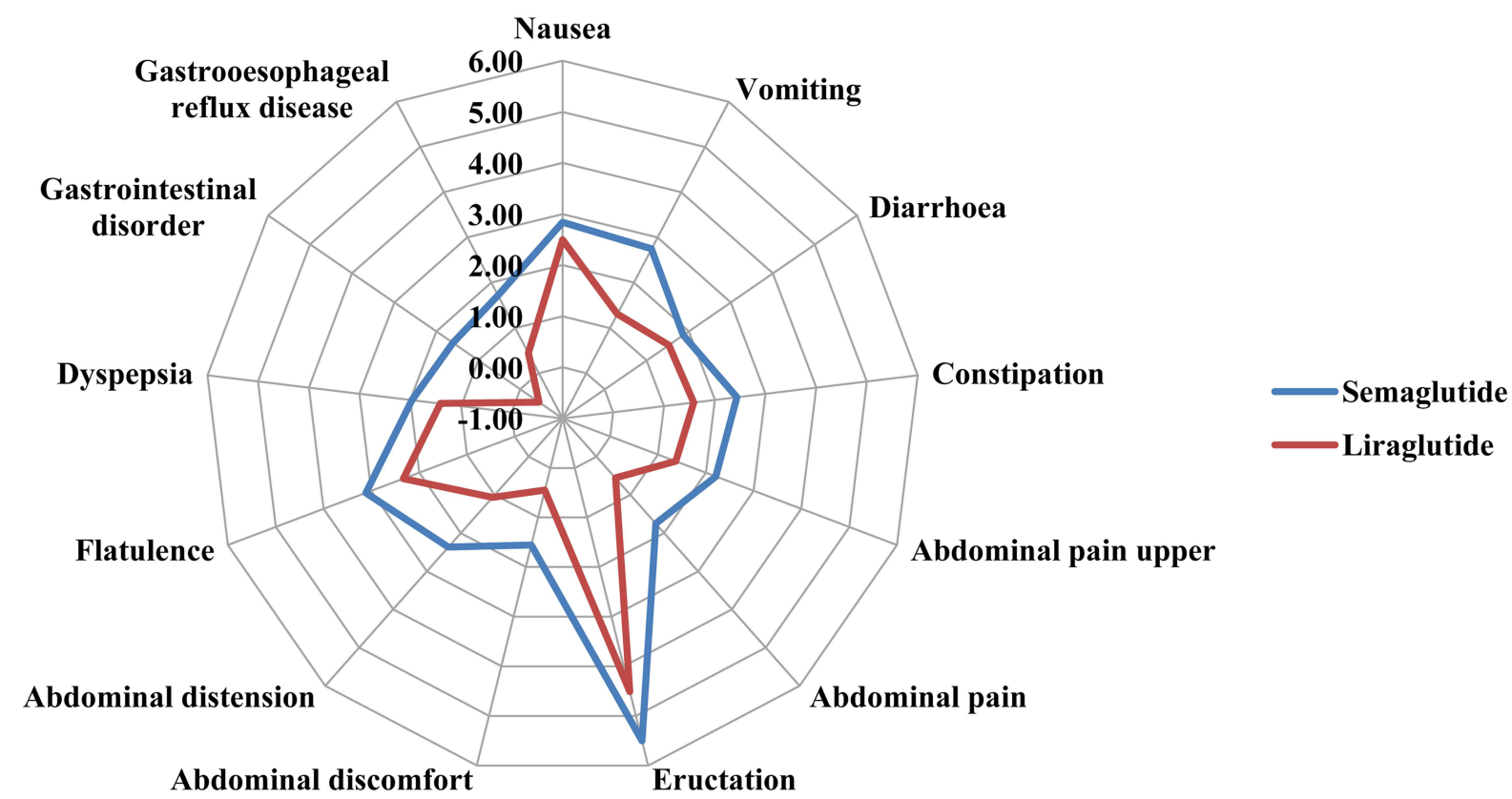

B

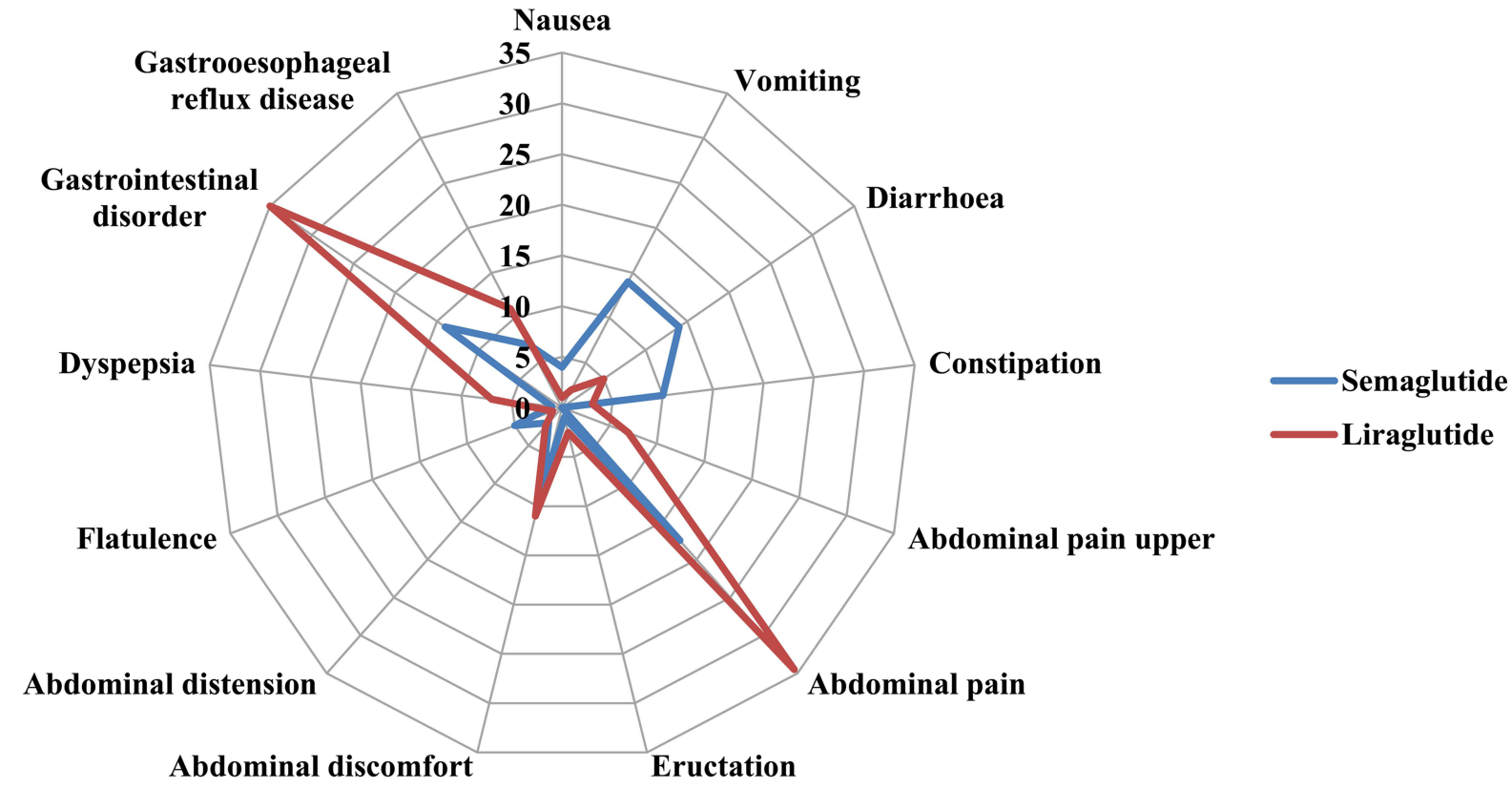

Figure I Differences in reporting of gastrointestinal adverse drug reactions between different GLP-IRAs as a radar chart. (A) Reporting-risk profile. (B) Time-to-onset profile. RORs $(95 \% \mathrm{Cls})$ for reporting risk were calculated through a logarithmic transformation.

1RAs (or exercise combined with GLP-RAs) can provide an efficacious and well-tolerated treatment option to help people with obesity achieve and maintain weight targets. $^{18-20}$ Nevertheless, some clinical studies have revealed safety issues that should also be addressed. For example, the results of a network meta-analysis suggested that among the weight-loss drugs approved by the FDA, liraglutide was associated with the highest odds of adverse event-related treatment discontinuation compared with those of a placebo. ${ }^{3}$ Tasporutide (GLP-1RA to be taken QW) use was associated with an increased prevalence of hypersensitivity and GADRs. These events made tasporutide clinically unacceptable and, therefore, a phase-III clinical trial was terminated in September $2010 .^{21,22}$ 
Table 3 Time-to-Onset of Cases with Semaglutide- or Liraglutide-Associated Gastrointestinal Adverse Drug Reactions

\begin{tabular}{|l|c|c|c|c|}
\hline \multirow{2}{*}{$\begin{array}{l}\text { Gastrointestinal Adverse Drug } \\
\text { Reaction }\end{array}$} & \multicolumn{2}{|c|}{ Semaglutide } & \multicolumn{2}{c|}{ Liraglutide } \\
\cline { 2 - 5 } & $\begin{array}{c}\text { Cases, } \\
\text { Number }\end{array}$ & $\begin{array}{c}\text { Time-to-Onset, Days, IQR } \\
\text { (QI-Q3) }\end{array}$ & $\begin{array}{c}\text { Cases, } \\
\text { Number }\end{array}$ & $\begin{array}{c}\text { Time-to-Onset, Days IQR } \\
\text { (Q I-Q3) }\end{array}$ \\
\hline Nausea & 617 & $4(0-38)$ & 524 & $1(0-20)$ \\
Vomiting & 412 & $14(0-53)$ & 274 & $2(0-17.75)$ \\
Diarrhea & 357 & $14(0-54)$ & 279 & $5(0-28.5)$ \\
Constipation & 157 & $10(0-63)$ & 89 & $3(0-16)$ \\
Abdominal pain upper & 119 & $0(0-11)$ & 100 & $7(0-52)$ \\
Abdominal pain & 106 & $17.5(0-59)$ & 42 & $34.5(4.25-89)$ \\
Eructation & 76 & $1(0-25.75)$ & 34 & $2.5(0-20)$ \\
Abdominal discomfort & 80 & $8(0-40.75)$ & 40 & $11(0.5-71.5)$ \\
Abdominal distension & 63 & $2(0-30.5)$ & 39 & $2.5(0-19.5)$ \\
Flatulence & 61 & $5(0-30)$ & 9 & $1(0-12)$ \\
Dyspepsia & 51 & $1(0-25)$ & 27 & $7(0-54.5)$ \\
Gastrointestinal disorder & 18 & $14(0-30.25)$ & 940 & $35(13-219)$ \\
Gastroesophageal reflux disease & 39 & $7(0-38)$ & $11(0.5-71.5)$ \\
Pooled & 1061 & $7(0-48)$ & $4(0-34.5)$ \\
\hline
\end{tabular}

Notes: ${ }^{a}$ Cases with corresponding information that enabled the time-to-onset profile to be calculated. ${ }^{\mathrm{b}} \mathrm{A}$ report may have one or more gastrointestinal adverse drug reactions.

Hence, real-world observational studies are essential for providing complementary information (especially about ADRs) to randomized clinical trials (RCTs) because they involve a large number of patients in different populations in a real-world environment, rather than homogeneous patient groups participating in RCTs.

In studies of GLP-1RA therapy in patients with T2DM, the most frequently reported ADRs tend to arise from the gastrointestinal system, with nausea, vomiting, and diarrhea occurring in up to $51 \%, 19 \%$, and $20 \%$ of patients, respectively. ${ }^{12}$ These GADRs are thought to be related to the inhibition effect of GLP1-RAs on gastrointestinal motility and stimulation of neural circuitry. ${ }^{23-26}$ Similarly, in our study, irrespective of semaglutide and liraglutide, the number of reported cases of nausea, vomiting, and diarrhea was the highest. However, semaglutide had a higher pooled
ROR and later pooled time-to-onset median value of GADRs compared with those for liraglutide. The reportingrisk profile and time-to-onset profile of assigned GADRs between semaglutide and liraglutide carried a significant difference. These findings are consistent with some current evidence. A systematic analysis of published clinical trials revealed important differences among the same subclass of long-acting GLP-1RAs (dulaglutide, albiglutide, exenatide (QW), liraglutide) which determined the risk of nausea, vomiting, and diarrhea. ${ }^{25}$ Another clinical study reported that semaglutide had more frequent GADRs and ADRs leading to premature discontinuation of treatment compared with those of liraglutide $(43.9 \%$ vs $38.3 \%$, and $11.4 \%$ vs $6.6 \%$, respectively). ${ }^{27}$ The same clinical study detailed a comparison of semaglutide with another long-acting GLP-1RA (exenatide (QW)). ${ }^{28}$ Such differential reporting-
A

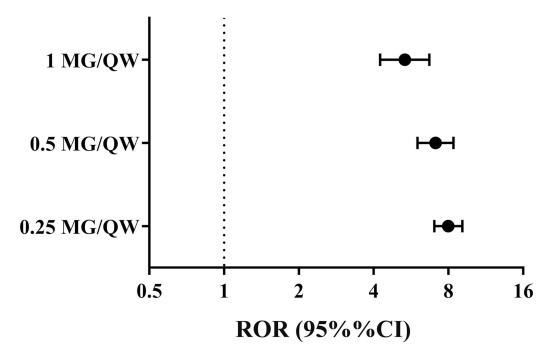

B

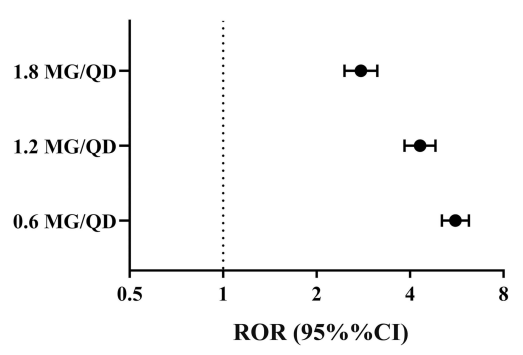

Figure 2 Reporting risk for gastrointestinal adverse drug reactions with GLP-IRAs grouped by subcutaneous dose. (A) Semaglutide. (B) Liraglutide. RORs (95\% Cls) were calculated through a logarithmic transformation.

Abbreviations: $\mathrm{ROR}$, reporting odds ratio; $\mathrm{Cl}$, confidence interval. 
risk and time-to-onset profiles of GADRs may have been dependent upon the pharmacokinetic and pharmacodynamic properties of the two GLP-1RAs. Semaglutide represents a new generation of long-acting GLP-1RAs with a longer half-life than liraglutide after subcutaneous administration (183 vs $11-15$ h). ${ }^{29}$ Hence, the effects of gastrointestinal motility and neural circuitry will be more significant and longer-acting during long-term weight-loss treatment with semaglutide compared with those using liraglutide. The gastrointestinal tolerance of individuals to a subclass of long-acting GLP-1RAs may also illustrate this imbalance to a certain degree. There is evidence suggesting that GLP1-induced deceleration of gastric emptying can be subject to rapid tachyphylaxis at the level of vagal nervous activation. ${ }^{23}$ These differences in gastric emptying appear to translate into the clinical setting. One may speculate that unequal GADRs might be associated with tachyphylaxis development upon treatment with different long-acting GLP-1RAs: this hypothesis requires further research. However, the availability of GLP-1RAs with different GADR properties could increase the flexibility of individualized care to patients suffering from obesity while achieving the same weight-loss effect.

The GADRs associated with use of semaglutide or liraglutide were not reported at a significantly higher prevalence if employed at higher doses in our study: our data are not in accordance with results from clinical trials. ${ }^{25}$ Common GADRs (nausea, vomiting, diarrhea) occur during dose escalation, ${ }^{16}$ with higher doses being more efficacious but consistently associated with unacceptable tolerability, ${ }^{30,31}$ which can lead to premature discontinuation of treatment in some patients. Drug manufacturers have recommended a gradual escalation in the dose of semaglutide and liraglutide, starting at a low dose followed by weekly increments to reach the maximum therapeutic dose. Hence, dosing for liraglutide and semaglutide should be titrated initially. ${ }^{32}$ If a patient experiences obvious GADRs, dose escalation can be delayed; if a further incremental dose is not tolerated, but treatment effects are noted, maintenance at the lower tolerated dose may be preferable to treatment discontinuation. ${ }^{6,16}$ Hence, the GADRs associated with the use of semaglutide or liraglutide may be reported at a lower tolerated dose in a database providing spontaneous reporting.

The GADRs induced by GLP-1RAs are associated with compliance to long-term treatment for obesity, as well as the risk of withdrawal from clinical trials. ${ }^{25,33}$ Some strategies for drug discovery may be implemented to help manage/ mitigate potential GADRs if administering a GLP-1RA for treatment of overweight or obesity. One area of important future research with regard to developing more efficacious GLP-1-based weight-loss therapies is to dissociate the neural circuits mediating GLP-1R-associated anorectic effects from nausea and malaise. ${ }^{24}$ In addition, a recent study showed that modulation of GLP-1RA trafficking may achieve greater metabolic control without increasing the rate of unwanted GADRs (eg, nausea). ${ }^{31}$

There were three main limitations in our study, all of which were intrinsic to FAERS use. First, ADR reporting is voluntary, and comes from heterogeneous sources (eg, pharmaceutical enterprises, medical staff, lawyers, consumers), which increases the possibility of underreporting. Second, FAERS provides limited data on patient characteristics. Missing data on medical history, and inspection/progression of disease add to the difficulties of assessment of ADR risk due to the limited ability to control confounders. Third, a case/non-case study cannot provide definite proof of a causal relationship, and is based on overreporting but not excess risk. We could not determine which GLP-1RA was more likely to cause GADRs. We could only list GLP-1RAs for assigned GADRs was more overreported, which is an indirect approach to determine GADR risk. Unfortunately, the denominator of drug exposure is absent in FAERS, so the prevalence of GADRs associated with GLP-1RA use cannot be estimated using FAERS.

\section{Conclusion}

Overall, the results of this real-world study support the concerns regarding GADRs if using semaglutide or liraglutide for overweight or obesity. Of equal importance, the GADRs associated with these two GLP-1RAs showed a significant difference in the profiles of reporting risk and time-to-onset. Hence, attention should be paid to individualized-treatment choices when choosing an agent from this class.

\section{Acknowledgments}

The authors thank the US Food and Drug Administration Adverse Event Reporting System for using their database.

\section{Author Contributions}

All authors made substantial contributions to conception and design, acquisition of data, or analysis and interpretation of data; took part in drafting the article or revising it critically for important intellectual content; agreed to 
submit to the current journal; gave final approval of the version to be published; and agreed to be accountable for all aspects of the work.

\section{Funding}

This study was funded by the Starting Fund for Scientific Research of High-level Talents of Fujian Medical University (No. XRCZX2020003).

\section{Disclosure}

The authors declared no conflicts of interest, financial or otherwise, in this work.

\section{References}

1. Afshin A, Forouzanfar MH, Reitsma MB, et al. Health effects of overweight and obesity in 195 countries over 25 years. $N$ Engl J Med. 2017;377(1):13-27. doi:10.1056/NEJMoa1614362

2. Fujioka K, Harris SR. Barriers and solutions for prescribing obesity pharmacotherapy. Endocrinol Metab Clin North Am. 2020;49 (2):303-314. doi:10.1016/j.ecl.2020.02.007

3. Khera R, Murad MH, Chandar AK, et al. Association of pharmacological treatments for obesity with weight loss and adverse events: a systematic review and meta-analysis. JAMA. 2016;315 (22):2424-2434. doi:10.1001/jama.2016.7602

4. Andersen A, Lund A, Knop FK, Vilsbøll T. Glucagon-like peptide 1 in health and disease. Nat Rev Endocrinol. 2018;14(7):390-403. doi:10.1038/s41574-018-0016-2

5. Lehmann EW, Torekov SS. Glucagon-like peptide-1 receptor agonists: the key to healthy weight loss maintenance? Cardiovasc Res. 2021;117(10):e120-e122. doi:10.1093/cvr/cvab249

6. Jph W, R1 B, Calanna S, et al. Once-weekly semaglutide in adults with overweight or obesity. $N$ Engl J Med. 2021;384(11):989. doi:10.1056/NEJMoa2032183

7. Wadden TA, Bailey TS, Billings LK, et al. Effect of subcutaneous semaglutide vs placebo as an adjunct to intensive behavioral therapy on body weight in adults with overweight or obesity: the STEP 3 randomized clinical trial. JAMA. 2021;325(14):1403-1413. doi:10.1001/jama.2021.1831

8. Rubino D, Abrahamsson N, Davies M, et al. Effect of continued weekly subcutaneous semaglutide vs placebo on weight loss maintenance in adults with overweight or obesity: the STEP 4 randomized clinical trial. JAMA. 2021;325(14):1414-1425. doi:10.1001/ jama.2021.3224

9. Christensen RM, Juhl CR, Torekov SS. Benefit-risk assessment of obesity drugs: focus on glucagon-like peptide-1 receptor agonists. Drug Saf. 2019;42(8):957-971. doi:10.1007/s40264-019-00812-7

10. Madsbad S. Review of head-to-head comparisons of glucagon-like peptide-1 receptor agonists. Diabetes Obes Metab. 2016;18 (4):317-332. doi:10.1111/dom.12596

11. Meier JJ. GLP-1 receptor agonists for individualized treatment of type 2 diabetes mellitus. Nat Rev Endocrinol. 2012;8(12):728-742. doi:10.1038/nrendo.2012.140

12. Filippatos TD, Panagiotopoulou TV, Elisaf MS. Adverse effects of GLP-1 receptor agonists. Rev Diabet Stud. 2014;11(3-4):202-230. Fall-Winter. doi:10.1900/rds.2014.11.202

13. Maciá-Martínez MA, de Abajo FJ, Roberts G, Slattery J, Thakrar B, Wisniewski AF. An empirical approach to explore the relationship between measures of disproportionate reporting and relative risks from analytical studies. Drug Saf. 2016;39(1):29-43. doi:10.1007/ s40264-015-0351-3
14. Liu C, Zou Y, Qian H. GLP-1R agonists for the treatment of obesity: a patent review (2015-present). Expert Opin Ther Pat. 2020;30 (10):781-794. doi:10.1080/13543776.2020.1811851

15. Aaseth J, Ellefsen S, Alehagen U, Sundfør TM, Alexander J. Diets and drugs for weight loss and health in obesity - an update. Biomed Pharmacother. 2021;140:111789. doi:10.1016/j.biopha.2021.111789

16. Ard J, Fitch A, Fruh S, Herman L. Weight loss and maintenance related to the mechanism of action of glucagon-like peptide 1 receptor agonists. Adv Ther. 2021;38(6):2821-2839. doi:10.1007/s12325021-01710-0

17. Grill HJ. A role for GLP-1 in treating hyperphagia and obesity. Endocrinology. 2020;161(8). doi:10.1210/endocr/bqaa093

18. Pi-Sunyer X, Astrup A, Fujioka K, et al. A randomized, controlled trial of $3.0 \mathrm{mg}$ of liraglutide in weight management. $N$ Engl J Med. 2015;373(1):11-22. doi:10.1056/NEJMoa1411892

19. Kushner RF, Calanna S, Davies M, et al. Semaglutide $2.4 \mathrm{mg}$ for the treatment of obesity: key elements of the STEP trials 1 to 5. Obesity. 2020;28(6):1050-1061. doi:10.1002/oby.22794

20. Lundgren JR, Janus C, Jensen SBK, et al. Healthy weight loss maintenance with exercise, liraglutide, or both combined. $N$ Engl J Med. 2021;384(18):1719-1730. doi:10.1056/NEJMoa2028198

21. Giraudon M, Sturm S, Lambert N, et al. Effect of varying degrees of renal impairment on the pharmacokinetics and tolerability of taspoglutide. Diabetes Obes Metab. 2017;19(4):537-544. doi: $10.1111 /$ dom. 12850

22. Rosenstock J, Balas B, Charbonnel B, et al. The fate of taspoglutide, a weekly GLP-1 receptor agonist, versus twice-daily exenatide for type 2 diabetes: the T-emerge 2 trial. Diabetes Care. 2013;36 (3):498-504. doi:10.2337/dc12-0709

23. Nauck MA, Kemmeries G, Holst JJ, Meier JJ. Rapid tachyphylaxis of the glucagon-like peptide 1-induced deceleration of gastric emptying in humans. Diabetes. 2011;60(5):1561-1565. doi:10.2337/db10-0474

24. Kanoski SE, Hayes MR, Skibicka KP. GLP-1 and weight loss: unraveling the diverse neural circuitry. Am J Physiol Regul Integr Comp Physiol. 2016;310(10):R885-R895. doi:10.1152/ajpregu.00520.2015

25. Bettge K, Kahle M, Abd El Aziz MS, Meier JJ, Nauck MA. Occurrence of nausea, vomiting and diarrhoea reported as adverse events in clinical trials studying glucagon-like peptide-1 receptor agonists: a systematic analysis of published clinical trials. Diabetes Obes Metab. 2017;19(3):336-347. doi:10.1111/dom.12824

26. Rehfeld JF, Knop FK, Asmar M. Gastrin secretion in normal subjects and diabetes patients is inhibited by glucagon-like peptide 1: a role in the gastric side effects of GLP-1-derived drugs? Scand J Gastroenterol. 2019;54(12):1448-1451. doi:10.1080/00365521.2019.1690673

27. Capehorn MS, Catarig AM, Furberg JK, et al. Efficacy and safety of once-weekly semaglutide $1.0 \mathrm{mg}$ vs once-daily liraglutide $1.2 \mathrm{mg}$ as add-on to 1-3 oral antidiabetic drugs in subjects with type 2 diabetes (SUSTAIN 10). Diabetes Metab. 2020;46(2):100-109. doi:10.1016/j. diabet.2019.101117

28. Ahmann AJ, Capehorn M, Charpentier G, et al. Efficacy and safety of once-weekly semaglutide versus exenatide ER in subjects with type 2 diabetes (SUSTAIN 3): a 56-week, open-label, randomized clinical trial. Diabetes Care. 2018;41(2):258-266. doi:10.2337/dc17-0417

29. Christou GA, Katsiki N, Blundell J, Fruhbeck G, Kiortsis DN. Semaglutide as a promising antiobesity drug. Obes Rev. 2019;20 (6):805-815. doi:10.1111/obr.12839

30. Barrington P, Chien JY, Showalter HD, et al. A 5-week study of the pharmacokinetics and pharmacodynamics of LY2189265, a novel, long-acting glucagon-like peptide-1 analogue, in patients with type 2 diabetes. Diabetes Obes Metab. 2011;13(5):426-433. doi:10.1111/ j.1463-1326.2011.01364.x

31. Nauck MA, Petrie JR, Sesti G, et al. A Phase 2, Randomized, Dose-Finding Study of the novel once-weekly human GLP-1 analog, semaglutide, compared with placebo and open-label liraglutide in patients with type 2 diabetes. Diabetes Care. 2016;39(2):231-241. doi: $10.2337 / \mathrm{dc} 15-0165$ 
32. Knudsen L, Lau J. The discovery and development of liraglutide and semaglutide. Front Endocrinol. 2019;10:155. doi:10.3389/fendo.2 019.00155
33. Nuffer WA, Trujillo JM. Liraglutide: a new option for the treatment of obesity. Pharmacotherapy. 2015;35(10):926-934. doi:10.1002/ phar.1639

\section{Publish your work in this journal}

Diabetes, Metabolic Syndrome and Obesity: Targets and Therapy is an international, peer-reviewed open-access journal committed to the rapid publication of the latest laboratory and clinical findings in the fields of diabetes, metabolic syndrome and obesity research. Original research, review, case reports, hypothesis formation, expert opinion and commentaries are all considered for publication. The manuscript management system is completely online and includes a very quick and fair peer-review system, which is all easy to use. Visit $\mathrm{http}: / / \mathrm{www}$.dovepress.com/testimonials.php to read real quotes from published authors. 